\title{
Microsymposia
}

MS.72.1

Acta Cryst. (2008). A64, C123

Combining microfluidics with micro-SAXS for studies of the dynamics of DNA compaction

\section{Thomas Pfohl}

Max Planck Institute for Dynamics and Self-Organization, Bunsenstrasse 10, Goettingen, Niedersachsen, 37073, Germany, E-mail : thomas.pfohl@ ds.mpg.de

The miniaturization, integration, and analysis of chemical and biological processes on the nanoliter-scale have currently led to remarkable progress in biotechnology, protein crystallization, and combinatorial chemistry. Aside from having advantages such as reduction of sample volumes and shorter reaction and analysis times, microfluidics is a powerful tool for investigations of soft condensed matter and biological systems. The marriage of microfluidics with spatially resolved microdiffraction provides new opportunities to study time-resolved reaction dynamics as well as non-equilibrium dynamics of biomaterials. The characterization of these complex materials, which are typically liquid-crystalline at ambient conditions, is significantly improved owing to a concurrent orientation during the self assembly process. Moreover, the influence of external stress on the biological materials can be analyzed. Here, we present experiments on the dynamics of the compaction of DNA by linker-histone proteins and artificial polyimine dendrimers.

Keywords: microdiffraction, microfluidics, DNA-packing

\section{MS.72.2}

Acta Cryst. (2008). A64, C123

Application of SWAXS in nanomedicine:

Characterizing nanoparticles and their interaction with skin

Marianna Foldvari, Shawn Wettig

University of Waterloo, School of Pharmacy, Waterloo, Ontario, N2L 3G1, Canada, E-mail:foldvari@uwaterloo.ca

We employed small- and wide-angle X-ray scattering (SWAXS) to physicochemically characterize the structure of nanoparticles prepared from a range of gemini surfactants and plasmid for in vitro and in vivo DNA transfection studies. Gemini surfactants encapsulate plasmid DNA, creating an advanced type of nanoparticle-based delivery system (gemini NPs) designed to transfer exogenous DNA into tissues for subsequent expression of desired therapeutic protein. Gemini surfactants provide a basis to develop novel nonviral delivery systems for gene therapy; however, gemini NPs must possess several crucial properties to overcome difficult cellular and tissue barriers. For example, gemini surfactants must bind and condense DNA optimally. Then, the resulting gemini NP must transport DNA to the cell membrane surface, facilitate membrane translocation and endosome disruption, and ultimately localize the plasmid DNA in the nucleus. These crucial properties strongly depend on the structural features of the gemini NP. We found that the presence of mixed polymorphic structures in the gemini NP (lamellar, hexagonal, cubic phases) result in higher transfection efficiencies both in vitro and in vivo. SWAXS techniques were also used to examine the effects of gemini NPs on the human stratum corneum (SC) lipid barrier. The SC lipid barrier typically has two distinct lamellar structures, the long and short lamellar phases, with repeat spacings of approximately 13.4 and $6 \mathrm{~nm}$, respectively. The degree of disruption of the SC lipid barrier appears to be correlated with permeation into the skin. Therefore, monitoring the lipid barrier structure after treatment with traditional skin permeation enhancers and gemini NPs provide information on the skin delivery capacity of these pharmaceutical systems.

Keywords: X-ray scattering, gene therapy, transdermal

\section{MS.72.3}

Acta Cryst. (2008). A64, C123

\section{Peptidic nanotubes : From drug release to glass nanowires}

Franck Artzner $^{1}$, Emilie Pouget ${ }^{1}$, Christophe Tarabout ${ }^{1}$,

Celine Valery $^{1}$, Annie Cavalier ${ }^{1}$, Theyencheri Narayanan ${ }^{2}$,

Maite Paternostre ${ }^{3}$, Erik Dujardin ${ }^{4}$

${ }^{1}$ CNRS, Physics, franck.artzner@univ-rennes1.fr, Rennes, 35 Ile et Villaine, 35042 Rennes Cedex, France, ${ }^{2} \mathrm{ESRF}$, Grenoble, France, ${ }^{3} \mathrm{CEA}$ Saclay, Gif/Yvette, France, ${ }^{4}$ CEMES, Toulouse, CNRS, France, E-mail : franck.artzner@univ-rennes1.fr

Lanreotide molecules is a small peptide that self-assembles in water into highly monodisperse supramolecular nanotubes, the diameter and wall thickness of which are $244 \AA$ and $18 \AA$ respectively. [Valery, PNAS, 2003]. Noteworthy, these nanotubes exhibit a monthly drug release and are commercially available as autogel. Recently, following biomineralization principles, we show that the selfassembled nanotubes can be used as a template to produce micronlong, bilayered silica nanotubes having a monodisperse diameter of $29 \mathrm{~nm}$ (figure below). The nanotubes organize spontaneously into centimeter-size, highly ordered bundles [Pouget, Nat. Materials, 2007]. Furthermore, the formation mechanism was elucidated and was revealed to be comparable with expected growing mechanism of sea-sponge spicules. Both pharmaceutical and inorganic nanotubes were fully characterized using a range of techniques including optical and electron microscopy, as well as Small Angle X ray Scattering (SAXS), and Fiber Diffraction.

Figure : bilayered silica nanotubes having a monodisperse diameter of $29 \mathrm{~nm}$ observed by Transmission Electron Microscopy and SAXS.

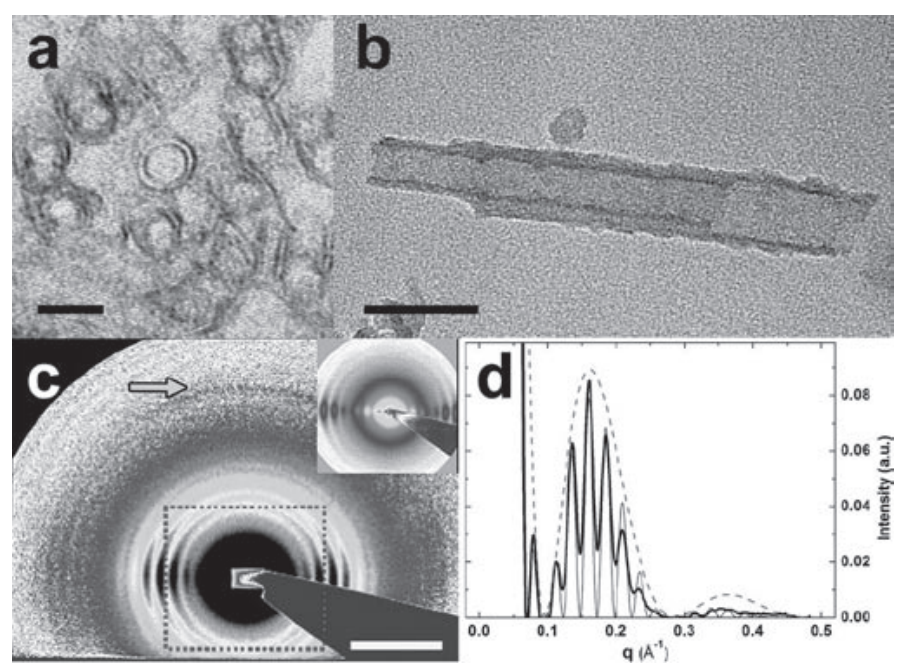

Keywords: SAXS, fibre diffraction, nanotubes 